\title{
On Logarithmic Convexity for Ky-Fan Inequality
}

\author{
Matloob Anwar' ${ }^{1}$ and J. Pečarić ${ }^{1,2}$ \\ ${ }^{1}$ Abdus Salam School of Mathematical Sciences, GC University, Lahore 54660, Pakistan \\ ${ }^{2}$ Faculty of Textile Technology, University of Zagreb, 10000 Zagreb, Croatia
}

Correspondence should be addressed to Matloob Anwar, matloob_t@yahoo.com

Received 19 November 2007; Accepted 14 February 2008

Recommended by Sever Dragomir

We give an improvement and a reversion of the well-known Ky-Fan inequality as well as some related results.

Copyright (C) 2008 M. Anwar and J. Pečarić. This is an open access article distributed under the Creative Commons Attribution License, which permits unrestricted use, distribution, and reproduction in any medium, provided the original work is properly cited.

\section{Introduction and preliminaries}

Let $x_{1}, x_{2}, \ldots, x_{n}$ and $p_{1}, p_{2}, \ldots, p_{n}$ be real numbers such that $x_{i} \in[0,1 / 2], p_{i}>0$ with $P_{n}=$ $\sum_{i=1}^{n} p_{i}$. Let $G_{n}$ and $A_{n}$ be the weighted geometric mean and arithmetic mean, respectively, defined by $G_{n}=\left(\prod_{i=1}^{n} x_{i}^{p_{i}}\right)^{1 / P_{n}}$, and $A_{n}=\left(1 / P_{n}\right) \sum_{i=1}^{n} p_{i} x_{i}=\bar{x}$. In particular, consider the abovementioned means $G_{n}^{\prime}=\left(\prod_{i=1}^{n}\left(1-x_{i}\right)^{p_{i}}\right)^{1 / P_{n}}$, and $A_{n}^{\prime}=\left(1 / P_{n}\right) \sum_{i=1}^{n} p_{i}\left(1-x_{i}\right)$. Then the wellknown Ky-Fan inequality is

$$
\frac{G_{n}}{G_{n}^{\prime}} \leq \frac{A_{n}}{A_{n}^{\prime}}
$$

It is well known that Ky-Fan inequality can be obtained from the Levinson inequality [1], see also [2, page 71$]$.

Theorem 1.1. Let $f$ be a real-valued 3-convex function on $[0,2 a]$, then for $0<x_{i}<a, p_{i}>0$,

$$
\frac{1}{P_{n}} \sum_{i=1}^{n} p_{i} f\left(x_{i}\right)-f\left(\frac{1}{P_{n}} \sum_{i=1}^{n} p_{i} x_{i}\right) \leq \frac{1}{P_{n}} \sum_{i=1}^{n} p_{i} f\left(2 a-x_{i}\right)-f\left(\frac{1}{P_{n}} \sum_{i=1}^{n} p_{i}\left(2 a-x_{i}\right)\right) .
$$

In [3], the second author proved the following result. 
Theorem 1.2. Let $f$ be a real-valued 3-convex function on $[0,2 a]$ and $x_{i}(1 \leq i \leq n) n$ points on $[0,2 a]$, then

$$
\frac{1}{P_{n}} \sum_{i=1}^{n} p_{i} f\left(x_{i}\right)-f\left(\frac{1}{P_{n}} \sum_{i=1}^{n} p_{i} x_{i}\right) \leq \frac{1}{P_{n}} \sum_{i=1}^{n} p_{i} f\left(a+x_{i}\right)-f\left(\frac{1}{P_{n}} \sum_{i=1}^{n} p_{i}\left(a+x_{i}\right)\right) .
$$

In this paper, we will give an improvement and reversion of Ky-Fan inequality as well as some related results.

\section{Main results}

Lemma 2.1. Define the function

$$
\varphi_{s}(x)= \begin{cases}\frac{x^{s}}{s(s-1)(s-2)}, & s \neq 0,1,2 \\ \frac{1}{2} \log x, & s=0, \\ -x \log x, & s=1, \\ \frac{1}{2} x^{2} \log x, & s=2 .\end{cases}
$$

Then $\phi_{s}^{\prime \prime \prime}(x)=x^{s-3}$, that is, $\varphi_{s}(x)$ is 3-convex for $x>0$.

Theorem 2.2. Define the function

$$
\xi_{s}=\frac{1}{P_{n}} \sum_{i=1}^{n} p_{i}\left(\varphi_{s}\left(2 a-x_{i}\right)-\varphi_{s}\left(x_{i}\right)\right)-\varphi_{s}(2 a-\bar{x})+\varphi_{s}(\bar{x})
$$

for $x_{i}, p_{i}$ as in (1.2). Then

(1) for all $s, t \in I \subseteq R$,

$$
\xi_{s} \xi_{t} \geq \xi_{r}^{2}=\xi_{(s+t) / 2}^{2}
$$

that is, $\xi_{s}$ is log convex in the Jensen sense;

(2) $\xi_{s}$ is continuous on $I \subseteq R$, it is also $\log$ convex, that is, for $r<s<t$,

$$
\xi_{s}^{t-r} \leq \xi_{r}^{t-s} \xi_{t}^{s-r}
$$

with

$$
\xi_{0}=\frac{1}{2} \ln \left(\frac{G_{n}^{a} A_{n}}{G_{n} A_{n}^{a}}\right)
$$

where $G_{n}^{a}=\left(\prod_{i=1}^{n}\left(2 a-x_{i}\right)^{p_{i}}\right)^{1 / P_{n}}, A_{n}^{a}=\left(1 / P_{n}\right) \sum_{i=1}^{n} p_{i}\left(2 a-x_{i}\right)$. 
Proof. (1) Let us consider the function

$$
f(x, u, v, r, s, t)=f(x)=u^{2} \varphi_{s}(x)+2 u v \varphi_{r}(x)+v^{2} \varphi_{t}(x),
$$

where $r=(s+t) / 2, u, v, r, s, t$ are reals.

$$
f^{\prime \prime \prime}(x)=\left(u x^{s / 2-3 / 2}+v x^{t / 2-3 / 2}\right)^{2} \geq 0
$$

for $x>0$. This implies that $f$ is 3-convex. Therefore, by (1.2), we have $u^{2} \xi_{s}+2 u v \xi_{r}+v^{2} \xi_{t} \geq 0$, that is,

$$
\xi_{s} \xi_{t} \geq \xi_{r}^{2}=\xi_{(s+t) / 2}^{2}
$$

This follows that $\xi_{s}$ is log convex in the Jensen sense.

(2) Note that $\xi_{s}$ is continuous at all points $s=0, s=1$, and $s=2$ since

$$
\begin{aligned}
& \xi_{0}=\lim _{s \rightarrow 0} \xi_{s}=\frac{1}{2} \ln \left(\frac{G_{n}^{a} A_{n}}{G_{n} A_{n}^{a}}\right), \\
& \xi_{1}=\lim _{s \rightarrow 1} \xi_{s}=\frac{1}{P_{n}} \sum_{i=1}^{n} p_{i}\left(x_{i} \ln x_{i}-\left(2 a-x_{i}\right) \ln \left(2 a-x_{i}\right)\right)+(2 a-\bar{x}) \ln (2 a-\bar{x})-\bar{x} \ln \bar{x} \\
& \left.\xi_{2}=\lim _{s \rightarrow 2} \xi_{s}=\frac{1}{2}\left[\frac{1}{P_{n}} \sum_{i=1}^{n} p_{i}\left(\left(2 a-x_{i}\right)^{2} \ln \left(2 a-x_{i}\right)-x_{i}^{2} \ln x_{i}\right)\right)-(2 a-\bar{x})^{2} \ln (2 a-\bar{x})+\bar{x}^{2} \ln \bar{x}\right] .
\end{aligned}
$$

Since $\xi_{s}$ is a continuous and convex in Jensen sense, it is log convex. That is,

$$
(t-r) \ln \xi_{s} \leq(t-s) \ln \xi_{r}+(s-r) \ln \xi_{t},
$$

which completes the proof.

Corollary 2.3. For $x_{i}, p_{i}$ as in (1.2),

$$
1<\exp \left(2 \xi_{3}^{4} \xi_{4}^{-3}\right) \leq \frac{G_{n}^{a} A_{n}}{G_{n} A_{n}^{a}} \leq \exp \left(2 \xi_{-1}^{3 / 4} \xi_{3}^{1 / 4}\right) .
$$

Proof. Setting $s=0, r=-1$, and $t=3$ in Theorem 1.2 , we get $\xi_{0}^{4} \leq \xi_{-1}^{3} \xi_{3}$ or

$$
\xi_{0} \leq \xi_{-1}^{3 / 4} \xi_{3}^{1 / 4}
$$

Again setting $s=3, r=0$, and $t=4$ in Theorem 1.2 , we get $\xi_{3}^{4} \leq \xi_{0} \xi_{4}^{3}$ or

$$
\xi_{0} \geq \xi_{3}^{4} \xi_{4}^{-3}
$$

Combining both inequalities (2.12), (2.13), we get

$$
\xi_{3}^{4} \xi_{4}^{-3} \leq \xi_{0} \leq \xi_{-1}^{3 / 4} \xi_{3}^{1 / 4}
$$


Also we have $\xi_{s}$ positive for $s>2$; therefore, we have

$$
0<\xi_{3}^{4} \xi_{4}^{-3} \leq \xi_{0} \leq \xi_{-1}^{3 / 4} \xi_{3}^{1 / 4}
$$

Applying exponentional function, we get

$$
1<\exp \left(2 \xi_{3}^{4} \xi_{4}^{-3}\right) \leq \frac{G_{n}^{a} A_{n}}{G_{n} A_{n}^{a}} \leq \exp \left(2 \xi_{-1}^{3 / 4} \xi_{3}^{1 / 4}\right)
$$

Remark 2.4. In Corollary 2.3, putting $2 a=1$ we get an improvement of Ky-Fan inequality.

Theorem 2.5. Define the function

$$
\rho_{s}=\frac{1}{P_{n}} \sum_{i=1}^{n} p_{i}\left(\varphi_{s}\left(a+x_{i}\right)-\varphi_{s}\left(x_{i}\right)\right)-\varphi_{s}(a+\bar{x})+\varphi_{s}(\bar{x}),
$$

for $x_{i}, p_{i}$, a as for Theorem 1.1. Then

(1) for all $s, t \in I \subseteq R$,

$$
\rho_{s} \rho_{t} \geq \rho_{r}^{2}=\rho_{(s+t) / 2}^{2}
$$

that is, $\rho_{s}$ is log convex in the Jensen sense;

(2) $\rho_{s}$ is continuous on $I \subseteq R$, it is also $\log$ convex. That is for $r<s<t$,

$$
\rho_{s}^{t-r} \leq \rho_{r}^{t-s} \rho_{t}^{s-r}
$$

with

$$
\begin{gathered}
\rho_{0}=\frac{1}{2} \ln \left(\frac{\widetilde{G}_{n} A_{n}}{G_{n} \widetilde{A}_{n}}\right), \\
\text { where } \widetilde{G}_{n}=\left(\prod_{i=1}^{n}\left(a+x_{i}\right)^{p_{i}}\right)^{1 / P_{n}}, \tilde{A}_{n}=\left(1 / P_{n}\right) \sum_{i=1}^{n} p_{i}\left(a+x_{i}\right) .
\end{gathered}
$$

Proof. The proof is similar to the proof of Theorem 2.2.

Remark 2.6. Let us note that similar results for difference of power means were recently obtained by Simic in [4].

\section{References}

[1] N. Levinson, "Generalization of an inequality of Ky-Fan," Journal of Mathematical Analysis and Applications, vol. 8, no. 1, pp. 133-134, 1964.

[2] J. Pečarić, F. Proschan, and Y. L. Tong, Convex Functions, Partial Orderings, and Statistical Applications, vol. 187 of Mathematics in Science and Engineering, Academic Press, Boston, Mass, USA, 1992.

[3] J. Pečarić, "An inequality for 3-convex functions," Journal of Mathematical Analysis and Applications, vol. 90, no. 1, pp. 213-218, 1982.

[4] S. Simic, "On logarithmic convexity for differences of power means," Journal of Inequalities and Applications, vol. 2007, Article ID 37359, 8 pages, 2007. 